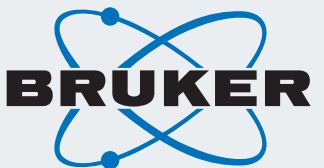

\title{
MALDI tissue imaging: mass spectrometric localization of biomarkers in tissue slices
}

New mass-spectrometric proteomics technologies have been developed with the potential to transition from pure research applications into the diagnostic laboratory. Of these, one very promising technique has already gained much interest in the research community: matrix-assisted laser desorption/ionization (MALDI) molecular imaging. For easy and successful application of MALDI imaging in the laboratory, Bruker Daltonics offers an innovative product line: a stand-alone automated sample preparation device, ImagePrep ${ }^{\mathrm{TM}}$; mass spectrometers with vital smartbeam ${ }^{\mathrm{TM}}$ laser technology: namely, the autoflex $\mathrm{III}^{\mathrm{TM}}$ and ultraflex III ${ }^{\text {TM }}$ MALDI-tandem time-of-flight (MALDI-TOF/TOF) instruments; and sophisticated software tools for fast data evaluation and biomarker visualization, flexlmaging ${ }^{\mathrm{T}}$.

Today, mass-spectrometric imaging is already revealing how proteins, peptides and even metabolites are spatially distributed in human and animal tissue slices by acquiring mass spectra across selected tissue areas, using a top-of-the-line dedicated laser (smartbeam). A twodimensional optical image of each tissue slice reveals its distribution of proteins, peptides or drug molecules. With the flexImaging software, the user can select several mass values and stain the tissue image with mass-specific colors (Fig. 1). Each spot on the color-coded tissue image is easily clickable for detailed analysis of the data supporting that colorcoded spot.

Substantial progress in imaging applications has recently been made with new mass-spectrometric technology ${ }^{1}$ (smartbeam laser, Bruker Daltonics), analysis tools (flexlmaging software, Bruker Daltonics) and even statistical analysis tools (ClassImaging from Bruker Daltonics; Fig. 1d).

\section{Tissue preparation for MALDI imaging}

Previously the bottleneck in MALDI imaging analysis has been sample preparation. The MALDI process requires that a matrix be applied to the sample. Present protocols suffer from various serious disadvantages, such as low robustness and poor reproducibility. Additionally, techniques such as pneumatic spraying or nanospotting are slow and have low spatial resolution.

The requests of scientists prompted Bruker Daltonics to develop a new matrix-application device for MALDI tissue imaging: the ImagePrep

Martin Schuerenberg, Christine Luebbert,

Sören-Oliver Deininger, Ralf Ketterlinus \& Detlev Suckau

Bruker Daltonik GmbH, Fahrenheitstrasse 4, 28359 Bremen, Germany. Correspondence should be addressed to D.S. (dsu@bdal.de).

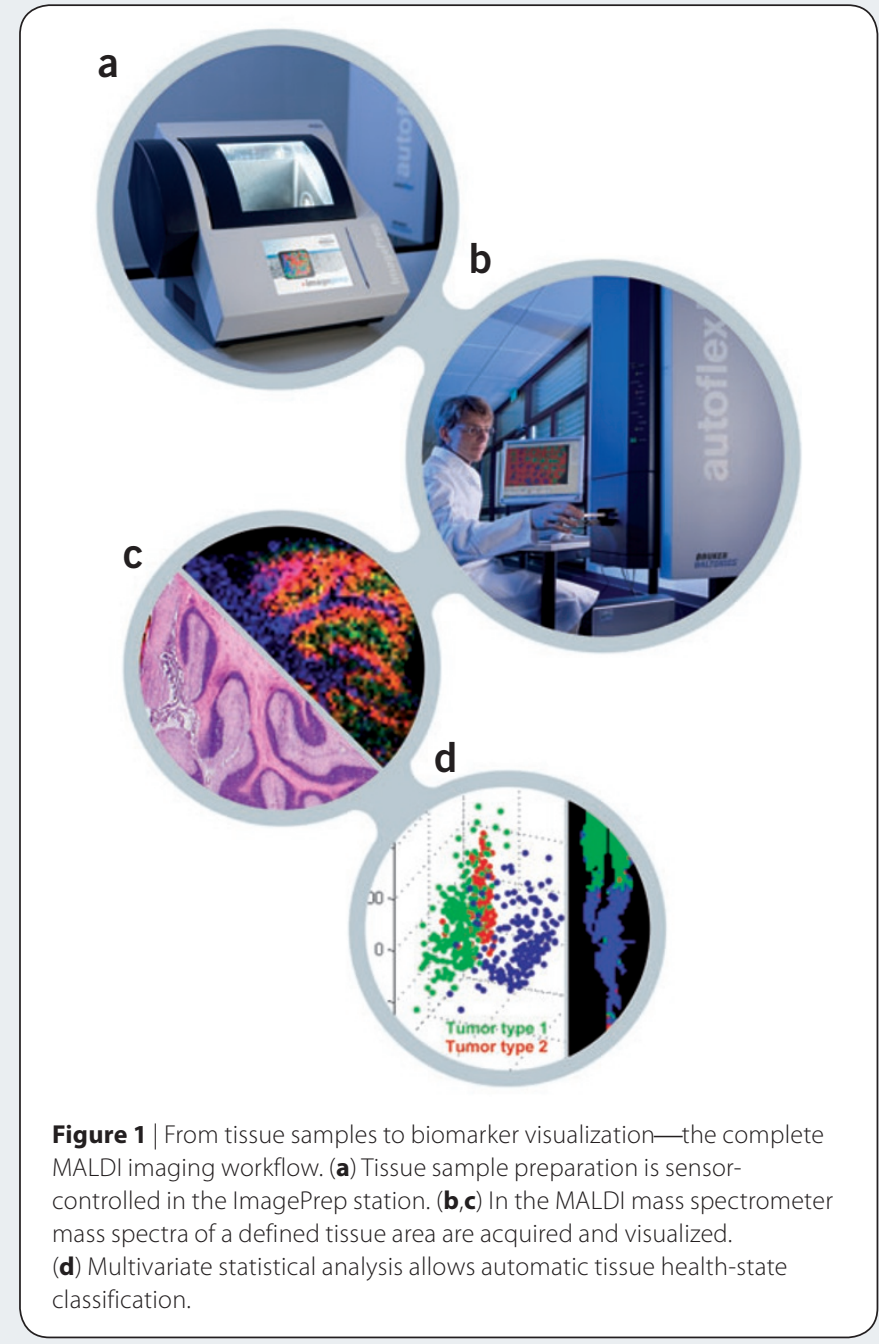


a

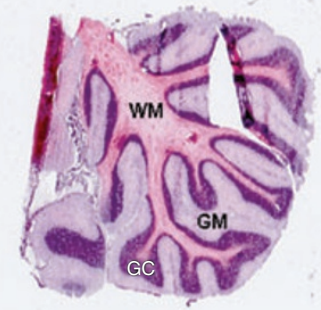

b
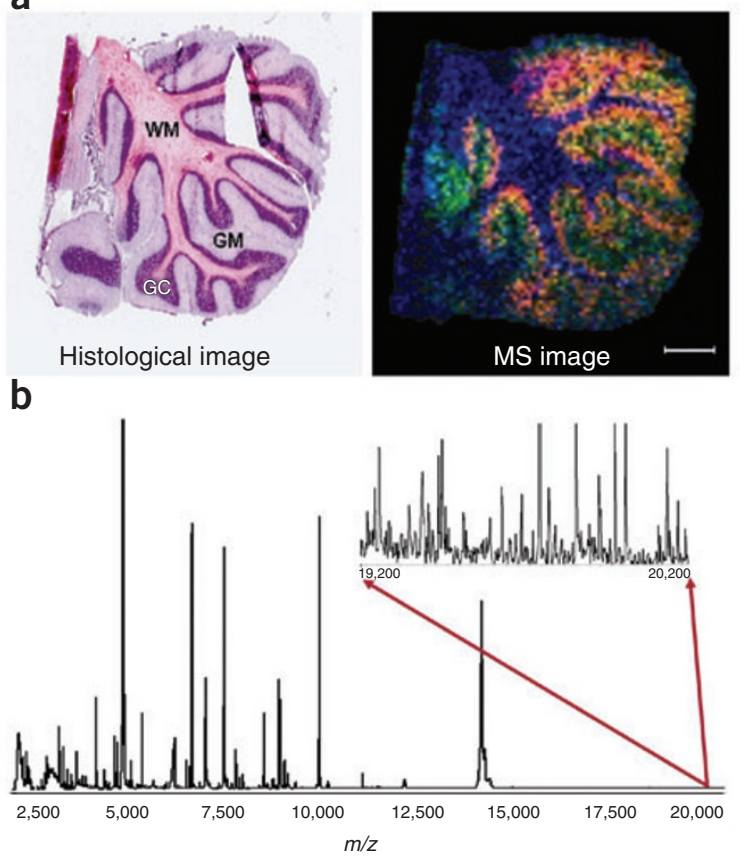

Figure $\mathbf{2}$ Analysis of a tissue slice of rat cerebellum. (a) The histological image (left) and the mass spectrometry image (right) of a tissue slice. Lateral image resolution is $70 \mu \mathrm{m}$. WM, white matter; GM, gray matter; GC, granule cells. Scale bar, $1 \mathrm{~mm}$. (b) The sum spectrum of all positions showed $>700$ individual peaks. Inset, higher resolution of the acquired spectrum.

station introduces preparation quality control for maximum performance at highest reproducibility in a push-button process (Fig. 1a).

The quality of MALDI tissue imaging analyses is dependent on the matrix application protocol. ImagePrep provides highly reproducible matrix preparations for MALDI tissue imaging in a fully automated process. Bruker's advanced ImagePrep technique is a new approach that consistently provides high-resolution images at high speed with superb spectral quality, without user intervention.

\section{Gentle matrix application by ImagePrep}

In the ImagePrep station, a matrix aerosol is created by vibrational vaporization under controlled conditions. It is then gently deposited onto tissue sections.

\section{Real-time preparation quality control}

An optical sensor monitors scattered light from the matrix layer, allowing the control of all relevant preparation parameters in real time. This quality control process is built into the preparation cycles and provides high reproducibility over several slides and on different days. User intervention is not required in the fully automated process.

\section{Acquisition of high-quality spectra}

Typically, images of tissues prepared in the ImagePrep station have an unmatched high spectrum quality with many peptide or protein
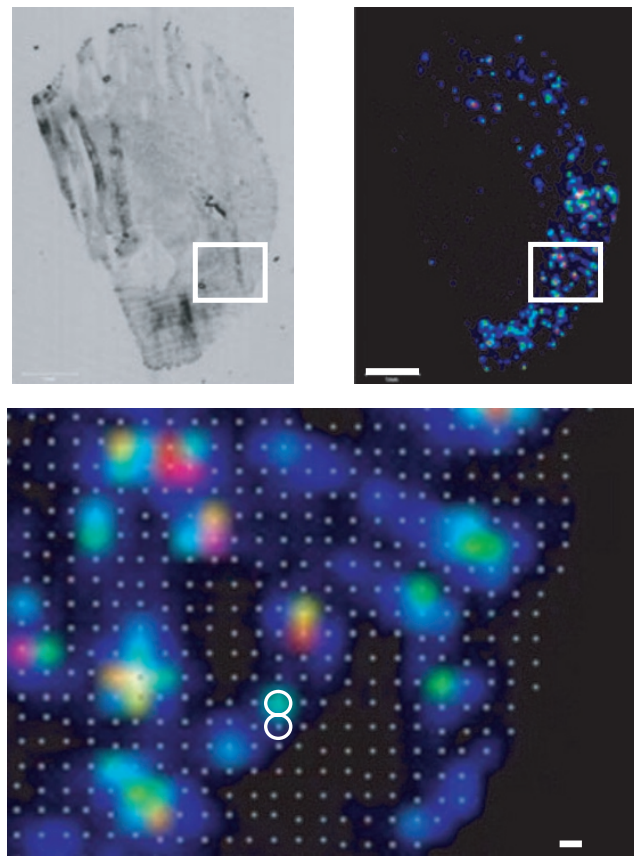

Figure 3 Amyloid plaques in rat brain. Optical image (top left) and MALD images (top right and bottom) of $\beta$-amyloid peptides. A lateral image resolution of $50 \mu \mathrm{m}$ was observed for different markers in this Alzheimer rat model. The boxes indicate the zoom range and the colored spots indicate the plaques with high intensity $\beta$-amyloid peptides. Scale bars, 1 mm (top) and $50 \mu \mathrm{m}$ (bottom).

signals, and a high spatial resolution comparable to that of images of tissues prepared by pneumatic spraying (Fig. 2). We observed a lateral resolution of $50 \mu \mathrm{m}$ in an Alzheimer rat model (Fig. 3).

\section{ImagePrep completes the MALDI molecular imager suite}

The ImagePrep station completes the MALDI Imaging solution from Bruker Daltonics, consisting of a high-performance MALDI-TOF/TOF system with $200-\mathrm{Hz}$ smartbeam laser technology and superb mass resolution delivered with PAN ${ }^{\top M}$ technology, an electrically conductive glass slide assembly, the most advanced flexImaging software delivering full integration from data acquisition to image analysis, and ClassImaging for tissue classification. Bruker's ClassImaging allows the automatic classification of tissue health states using multivariate statistical analysis of tissue biomarkers.

\section{ACKNOWLEDGMENTS}

We thank M. Riemenschneider (Laboratory of Neurochemistry and Neurogenetics, Department of Psychiatry and Psychotherapy, Klinikum Rechts der Isar, Munich, Germany) for the Alzheimer model brain sections.

1. Holle, A., Haase, A., Kayser, M. \& Hoehndorf, J. Optimizing UV laser focus profiles for improved MALDI performance. J. Mass Spectrom. 41, 705-716 (2006)

This article was submitted to Nature Methods by a commercial organization and has not been peer reviewed. Nature Methods takes no responsibility for the accuracy or otherwise of the information provided. 